\title{
Hartmann and Reynolds Numbers Effects in the Newtonian Blood Flow of a Bifurcated Artery with an Overlapping Stenosis
}

\author{
Norliza Mohd Zain and Zuhaila Ismail* \\ Department of Mathematical Sciences, Faculty of Science, Universiti Teknologi Malaysia \\ 81310 Johor Bahru, Malaysia \\ *Corresponding author: zuhaila@utm.my
}

Article history

Received: 25 March 2019

Received in revised form: 25 March 2019

Accepted: 25 May 2019

Published online: 1 August 2019

\begin{abstract}
Abstract Blood flow through a bifurcated artery with the presence of an overlapping stenosis located at parent's arterial lumen under the action of a uniform external magnetic field is studied in this paper. Blood is treated as an electrically conducting fluid which exhibits the Magnetohydrodynamics principle and it is characterized by a Newtonian fluid model. The governing equations are discretized using a stabilization technique of finite element known as Galerkin least-squares. The maximum velocity and pressure drop evaluated in this present study are compared with the results found in previous literature and COMSOL Multiphysics. The solutions found in a satisfactory agreement, thus verify the source code is working properly. The effects of dimensionless parameters of Hartmann and Reynolds numbers in the fluid's velocity and pressure are examined in details with further scientific discussions.
\end{abstract}

Keywords Magnetohydrodynamics; Newtonian; Galerkin least-squares; overlapping stenosis; bifurcated artery

Mathematics Subject Classification 76D05, 76M10, 76W05.

\section{Introduction}

Atherosclerosis is a key occurrence in cardiovascular disease and is the leading cause of mortality among humans worldwide [1,2]. This fact has been verified by World Health Organization (WHO) where in 2005, 17.5 million people have died from cardiovascular diseases [1]. In fact, this number is expected to grow to 23.3 million deaths by 2030 [2]. Atherosclerosis or in medical terms, stenosis consist of the deposition of cholesterol, cellular waste, fatty substance, fibrin and calcium on the inner lining of the artery $[3,4]$. This results in the narrowing of the blood vessel that may lead to inadequate and abnormal blood flow to organs and tissues [5]. As the flow of blood vessels supplying the heart muscle and brain are fully obstructed, heart attacks and strokes usually occur [2].

Static magnetic field generated by permanent magnets have been widely utilized in most medical devices to treat cardiovascular diseases rather than time varying electromagnetic field [2]. 
The production of Lorentz force according to the Lenz law opposing the fluid's motion provide a lot of medical benefits by controlling the flow of blood using magnetic field [6]. The retardation on the velocity of blood is proven useful for the treatment of diseases with an accelerated blood flow likes hypertension and haemorrhages [2]. In addition, the flow of blood influenced by an external magnetic field assist in reducing the bleeding during surgical procedures, decrease the healing time of fractures and effectiveness for the treatment of depression $[2,7]$. The strength of Lorentz force produced as the electrical conducting fluid interacts with an external magnetic field applied depends on the magnetic field intensity. It was found by applying magnetic flux intensity that exceeds $0.05 \mathrm{~T}$ can lead to an irreversible changes and heart fibrillation [2]. Whereas, a $0.4 \mathrm{~T}$ static magnetic field causes the changes in calcium dynamics that may also alter skin blood flow of individual humans [6]. Hence, this study concerns on the effects of Hartmann number, $M$ (a dimensionless parameter that appears due to the nondimensionalization procedures) that represent the strength of magnetic field to understand its contribution to haemodynamic factors of stenotic blood flow. Another dimensionless parameter that is also essential in most stenotic blood flow study is Reynolds number, Re that represent the ratio of the inertial force to the shearing force of the fluid. In other words, the Reynolds number could define the flow conditions which are either leading to laminar or turbulent flow corresponding to the arterial geometry [8]. It is believed that higher Reynolds number tends to develop more recirculation and enhance the shear stress magnitudes at the tightest occlusion which may severely damage the endothelial cells [5]. The malfunction of endothelial cells may reduce the lumen ability and influence the progress of cardiovascular diseases in the arteries [1].

Atherosclerosis preferentially develop at large and medium arteries with curvatures, junctions and bifurcations $[9,10]$ due to the sudden change of section area and curvature [11]. Realizing the impacts of complex geometry of an artery with the presence of stenosis to the blood flow dynamics, this study will pay attention on a bifurcated artery with an overlapping shaped stenosis. It is opined that by considering this type of geometry, the problem would become more acute since overlapping stenosis is usually formed at pulmonary and femoral arteries [6]. It is well known that the assumption of Newtonian behaviour for blood is valid when the flows is characterised by high shear rates of more than $100 \mathrm{~s}^{-1}$ flowing through a large vessel [12]. On the other hand, when the blood is flowing through a smaller or diseased vessel, those assumption breaks since non-Newtonian behaviour is more evident at low shear rates region $[8,12]$. According to [13], this variation of viscosity between Newtonian and non-Newtonian models is very small with the influence of an applied magnetic field. Thus, assuming the rheological behaviour of blood in this study as a Newtonian fluid would be acceptable.

Concerning on the influence of magnetic field as well as the domination of either inertial or viscous forces, the effects of two important dimensionless parameters which are Hartmann and Reynolds numbers are respectively studied. The streaming blood is assumed to behave according to the Newtonian fluid nature in a bifurcated channel with the presence of an overlapping shaped stenosis in parent's arterial lumen. The governing equations are decided to be solved by using one of the stabilization techniques of finite element method known as Galerkin-least squares due to its ability to circumvent from satisfying Babuska-Brezzi condition [14] which will be discussed further in Section 2 of this paper. 


\section{Mathematical Formulation}

\subsection{Arterial Geometry}

To demonstrate the mathematical analysis for the problem under consideration, these following assumptions are inflicted onto the geometry of an artery :

1. The artery forming bifurcation is of finite length.

2. The parent aorta possesses a single overlapping shaped stenosis in its lumen.

3. Curvatures are introduced at the lateral junctions and the flow divider of the arterial bifurcation to ensure that one can rule out the presence of any discontinuity causing non-existent of separation zones.

The geometry of the bifurcated artery with the presence of stenosis is modelled as a bifurcated channel as proposed by [15]. However, in this study an overlapping shaped stenosis described by [16] is considered instead of a mild shaped stenosis as shown in Figure 1.

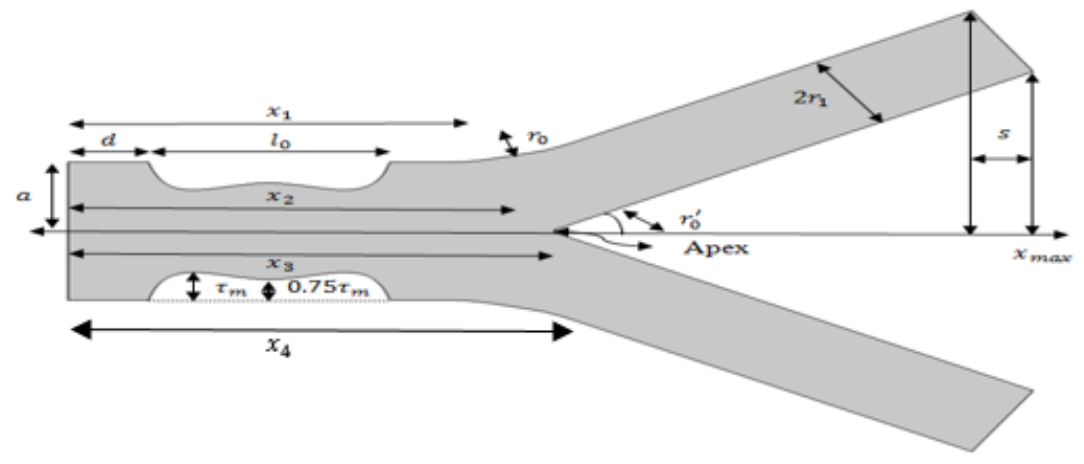

Figure 1: Geometry of the Stenosed Bifurcated Artery with an Overlapping Stenosis

The geometry of the bifurcated artery with the presence of an overlapping shaped stenosis may be set up mathematically of which the outer wall geometries are described by

$$
R_{1}(x)= \begin{cases}a, & 0 \leq x \leq d \text { and } d+l_{0} \leq x \leq x_{1}, \\
a-\frac{3 \tau_{m}}{2 l_{0}^{4}}\left\{\begin{array}{l}
11(x-d) l_{0}^{3}-47(x-d)^{2} l_{0}^{2} \\
+72(x-d)^{3} l_{0}-36(x-d)^{4}
\end{array}\right\}, & d \leq x \leq d+l_{0}, \\
a+r_{0}-\sqrt{r_{0}^{2}-\left(x-x_{1}\right)^{2}}, & x_{1} \leq x \leq x_{2}, \\
2 r_{1} \sec \beta+\left(x-x_{2}\right) \tan \beta, & x_{2} \leq x \leq x_{\max }-s .\end{cases}
$$

On the other hand, the inner wall geometries can be specified as

$$
R_{2}(x)= \begin{cases}0, & 0 \leq x \leq x_{3} \\ \sqrt{r_{0}^{\prime}-\left(x-\left(x_{3}+r_{0}^{\prime}\right)\right)^{2}}, & x_{3} \leq x \leq x_{4} \\ r_{0}^{\prime} \cos \beta+\left(x-x_{4}\right) \tan \beta, & x_{4} \leq x \leq x_{\max }\end{cases}
$$


where $R_{1}(x)$ and $R_{2}(x)$ represent the radii of the outer and inner wall, respectively. Meanwhile, $a$ and $r_{1}$ are the respective radii of the mother and daughter artery. $r_{0}$ and $r_{0}^{\prime}$ are the radii of curvature for the lateral junction and the flow divider, respectively. Whereas, $l_{0}$ is the length of the stenosis at a distance $d$ from the origin. Location of the onset and offset of the lateral junction are denoted by $x_{1}$ and $x_{2}$, respectively. $x_{3}$ indicates as the apex, $\tau_{m}$ represents the maximum height of stenosis occur at $d+2 l_{0} / 6$ and $d+4 l_{0} / 6$. While, $\beta$ denotes half of the bifurcation angle. Parameters involved in the above expressions (1) and (2) may be given as

$$
\begin{gathered}
x_{2}=x_{1}+r_{0} \sin \beta, r_{0}=\frac{a-2 r_{1} \sec \beta}{\cos \beta-1}, r_{0}^{\prime}=\frac{\left(x_{3}-x_{2}\right) \sin \beta}{1-\sin \beta}, \\
x_{3}=x_{2}+q, s=2 r_{1} \sin \beta,
\end{gathered}
$$

where $q$ is a small number lying in the range of $0.0001 \leq q \leq 0.0005$ chosen for the compatibility of the geometry and

$$
x_{4}=x_{3}+r_{0}^{\prime}(1-\sin \beta) \text {. }
$$

\subsection{Mathematical Model}

\subsubsection{Material Behaviour}

The relation between the stress and strain rate tensors are connected via non-linear relations given by a constitutive equation. According to $[17,18]$, by definition of the Generalized Newtonian Liquid (GNL) model, the stress tensor is the sum of volumetric stress and viscous stress components which can be written mathematically as

$$
\boldsymbol{\tau}=-p \boldsymbol{I}+2 \eta(\dot{\gamma}) \boldsymbol{D},
$$

where $\tau$ is the stress tensor, $p$ is the pressure or volumetric stress and $\boldsymbol{D}$ is the strain rate tensor which may be defined as

$$
\boldsymbol{D}=\frac{1}{2}\left(\nabla \boldsymbol{u}+(\nabla \boldsymbol{u})^{T}\right) .
$$

Whereas, $\eta$ is the viscosity function which is depending on the scalar $\dot{\gamma}$ that indicates the magnitude of the strain rate tensor given as

$$
\dot{\gamma}=\sqrt{2 D(\boldsymbol{u}): D(\boldsymbol{u})}
$$

GNL model allows the variation of viscosity with the shear rate. Thus, this model is useful in modelling the shearing flow and observing the effects of shear dominated flow under the natures of shear-thinning as well as shear-thickening [17]. However, this study only covers the Newtonian case where the viscosity is always constant with the shear rate, thus expression (6) is reduced to

$$
\boldsymbol{\tau}=-p \boldsymbol{I}+2 \mu \boldsymbol{D},
$$

where $\eta(\dot{\gamma})=\mu$ for such cases. Since this study is concerned on the effects of Hartmann and Reynolds numbers, thus by the non-dimensionalization procedure which involve the dimensionless variables below

$$
x=\frac{\bar{x}}{\bar{h}}, y=\frac{\bar{y}}{\bar{h}}, u=\frac{\bar{u}}{\bar{u}_{r}}, v=\frac{\widetilde{v}}{\overline{u_{r}}}, p=\frac{\bar{p}}{\bar{\rho} \bar{u}_{r}^{2}} .
$$


These two dimensionless parameters are respectively attained a

$$
M=B_{0}\left(\frac{\sigma h^{2}}{\mu}\right)^{1 / 2} \text { and } \operatorname{Re}=\frac{\rho u_{r} h}{\mu},
$$

where $B_{0}$ is the magnetic flux intensity, $\sigma$ is the electrical conductivity, $h$ is length of the inlet, $\mu$ represents the viscosity of the fluid, $\rho$ indicates the fluid's density, and $u_{r}$ is the flow's characteristic velocity.

\subsubsection{Governing Equations and Boundary Conditions}

The boundary value problem is constructed by incorporating the mass and momentum conservations as well as the GNL constitutive equation stated in equation (9). Hence, the governing equations with prescribed boundary conditions in a domain $\Omega \subset \Re^{2}$ can be written as follows

$$
\begin{array}{lr}
\nabla \cdot \boldsymbol{u}=0 & \text { in } \Omega, \\
\rho \boldsymbol{u} \cdot \nabla \boldsymbol{u}-\nabla \cdot(2 \eta(\dot{\gamma}) \boldsymbol{D})+\nabla p=\rho \boldsymbol{f} & \text { in } \Omega, \\
\boldsymbol{u}=\boldsymbol{u}_{g} & \text { on } \Gamma_{g}, \\
(-p \boldsymbol{I}+2 \eta(\dot{\gamma}) \boldsymbol{D}) \boldsymbol{n}=\boldsymbol{t}_{h} & \text { on } \Gamma_{h},
\end{array}
$$

where $\boldsymbol{u}$ is the velocity component, $\boldsymbol{f}$ is the body force vector, $\boldsymbol{u}_{g}$ is the prescribed boundary velocities, $\boldsymbol{n}$ is the unit outward normal vector, $\boldsymbol{I}$ is the second-order identity tensor and $\boldsymbol{t}_{h}$ is vector of the prescribed boundary tractions. In addition, $\Omega$ is the domain, $\Gamma$ is the boundary of the domain $\Omega$, while $\Gamma_{g}$ and $\Gamma_{h}$ denotes part of the boundary $\Gamma$ where Dirichlet and Neumann conditions are respectively imposed.

\section{Galerkin Least-Squares Formulation}

The appropriate weighting and trial functions for the velocity and pressure fields are approximated according to the usual approximation spaces for fluid dynamics as found in [17] and [18] given as,

$$
\begin{aligned}
& \boldsymbol{V}_{h}=\left\{\boldsymbol{N} \in H_{0}^{1}(\Omega)^{2} \mid \boldsymbol{N}_{\mid \mathrm{K}} \in R_{k}\left(\Omega_{K}\right)^{2}, \Omega_{K} \in C_{h}\right\}, \\
& \boldsymbol{V}_{h}^{g}=\left\{\boldsymbol{N} \in H^{1}(\Omega)^{2} \mid \boldsymbol{N}_{\mid \mathrm{K}} \in R_{k}\left(\Omega_{K}\right)^{2}, \Omega_{K} \in C_{h}, \boldsymbol{N}=\boldsymbol{u}_{g} \text { on } \Gamma_{g}\right\}, \\
& P_{h}=\left\{p \in C^{0}(\Omega) \cap L_{0}^{2}(\Omega) \mid p_{\mid \mathrm{K}} \in R_{1}\left(\Omega_{K}\right), \Omega_{K} \in C_{h}\right\} .
\end{aligned}
$$

The parameters $R_{k}$ and $R_{l}$ in equation (13) represent the polynomial spaces of degrees $k$ and $l$, respectively defined over a finite element partition $C_{h}$ of the problem domain $\bar{\Omega}$ consisting of triangular element $P_{1}$ parametrized by a characteristic mesh size $h_{K}$.

Based on the approximation functions defined in (13), Galerkin least-squares formulation for boundary value problem introduced in (12) can be stated as to find the pair $\left(\boldsymbol{u}_{h}, p_{h}\right) \in \boldsymbol{V}_{h}^{g} \times P_{h}$ such that

$$
B\left(\boldsymbol{u}_{h}, p_{h} ; \boldsymbol{N}, q\right)=F(\boldsymbol{N}, q), \forall(\boldsymbol{N}, q) \in\left(\boldsymbol{V}_{h} \times P_{h}\right),
$$


where

$$
\begin{aligned}
& B\left(\boldsymbol{u}_{h}, p_{h} ; \boldsymbol{N}, q\right)=\int_{\Omega} \rho \boldsymbol{u} \cdot \nabla \boldsymbol{u} \cdot \boldsymbol{N} d \Omega+\int_{\Omega} 2 \eta(\dot{\gamma}) \boldsymbol{D}(\boldsymbol{u}) \cdot \boldsymbol{D}(\boldsymbol{N}) d \Omega-\int_{\Omega} \rho \nabla \cdot \boldsymbol{N} d \Omega \\
& -\int_{\Omega} q \nabla \cdot \boldsymbol{u} d \Omega+\sum_{\Omega_{K \in C_{h}}} \int_{\Omega_{K}}\left[\begin{array}{l}
\left.\tau\left(R e_{K}\right)(\rho \boldsymbol{u} \cdot \nabla \boldsymbol{u}+\nabla p-2 \nabla \cdot(\eta(\dot{\gamma}) \boldsymbol{D}(\boldsymbol{u})))\right] d \Omega_{K}, \\
\cdot(\rho \boldsymbol{u} \cdot \nabla \boldsymbol{N}-\nabla q-2 \nabla \cdot(\eta(\dot{\gamma}) \boldsymbol{D}(\boldsymbol{u})))
\end{array}\right] \\
& F(\boldsymbol{N}, q)=\int_{\Omega} \rho \boldsymbol{f} \cdot \boldsymbol{N} d \Omega+\int_{\Gamma} \boldsymbol{t}_{h} \cdot \boldsymbol{N} d \Gamma \\
& +\sum_{\Omega_{K \in C_{h}}} \int_{\Omega_{K}}\left[\rho \boldsymbol{f} \cdot \tau\left(R_{K}\right)(\rho \boldsymbol{u} \nabla \cdot \boldsymbol{N}-\nabla q-2 \nabla \cdot(\eta(\dot{\gamma}) \boldsymbol{D}(\boldsymbol{v})))\right] d \Omega_{K} .
\end{aligned}
$$

The terms involved in equation (15) are counted elementwise with the positive parameter $\tau\left(R e_{K}\right)$ behaves as a stabilization parameter throughout this study defined according to [17] and [18] as follow

$$
\begin{aligned}
& \tau\left(R e_{K}\right)=\frac{h_{K}}{2|\boldsymbol{u}|_{2}} \xi\left(R e_{K}\right), \text { with } \\
& R e_{K}=\frac{m_{K} \rho|\boldsymbol{u}|_{2} h_{K}}{4 \eta(\dot{\gamma}) / \rho} \text { and }|\boldsymbol{u}|_{2}=\left(\sum_{i=1}^{2}\left|u_{i}\right|^{2}\right)^{1 / 2} \\
& \xi\left(R e_{K}\right)=\left\{R e_{K}, 0 \leq R e_{K}<11, R e_{K} \geq 1\right. \\
& \tau\left(R e_{K}\right)=\frac{h_{K}}{2|\boldsymbol{u}|_{2}} \xi\left(R e_{K}\right), \\
& m_{K}=\min \left\{1 / 3,2 C_{k}\right\}, \\
& h_{K}\|\Delta \boldsymbol{N}\|_{0, K}^{2} \leq C_{k}\|\nabla \boldsymbol{N}\|_{0}^{2}, \forall \boldsymbol{N} \in \boldsymbol{V}_{h} .
\end{aligned}
$$

The term $h_{K}$ acts as size of the triangular $K$-element. Equations (14)-(16) are discretized by expanding the trial functions $\boldsymbol{u}, p$ and weighting functions $\boldsymbol{N}, q$ in terms of its finite element shape functions. By transforming equation (15) into its residual form, $\mathbf{R}(\mathbf{U})$ that can be written as

$$
\mathbf{R}(\mathbf{U})=[\mathbf{K}(\mathbf{U})] \mathbf{U}-\{\mathbf{F}\}=0
$$

where $\mathbf{U}$ is vector for the degrees of freedom of $\boldsymbol{u}_{h}$ and $p_{h},[\mathbf{K}(\mathbf{U})]$ stands for the stiffness matrices and $\{\mathbf{F}\}$ denotes the force vector. To deal with the non-linearity presented in (15), the Newton-Raphson method is implemented where the solutions are found converged at $10^{-4}$. The convergence criteria $\tau$ of maximum residual norm is approximated by

$$
\tau \leq \sqrt{\sum_{I=1}^{N} R_{I}^{2}}
$$

Hence, according to the Newton-Raphson method the solutions for the degrees of freedom are found as

$$
\mathbf{U}^{b+1}=\mathbf{U}^{b}-\mathbf{J}^{-1}\left(\mathbf{U}^{b}\right) \mathbf{R}\left(\mathbf{U}^{b}\right),
$$


where $\mathbf{J}=\partial \mathbf{R} / \partial \mathbf{U}$, that is also known as the Jacobian matrix and $b$ is the iteration. Meanwhile, to approximate the integrals in equation (15), the Gaussian quadrature techniques are applied using six quadrature points throughout this study.

\section{Numerical Validation}

For the validation purpose of the source code that has been developed according to the GLS algorithms, an implementation into the Newtonian blood flow in a straight rectangular channel are carried out using unstructured triangular mesh of $21060 P_{1} / P_{1}$ elements with 10841 nodes. The computational domain involved in this validation step is demonstrated in Figure 2 as proposed by [19].

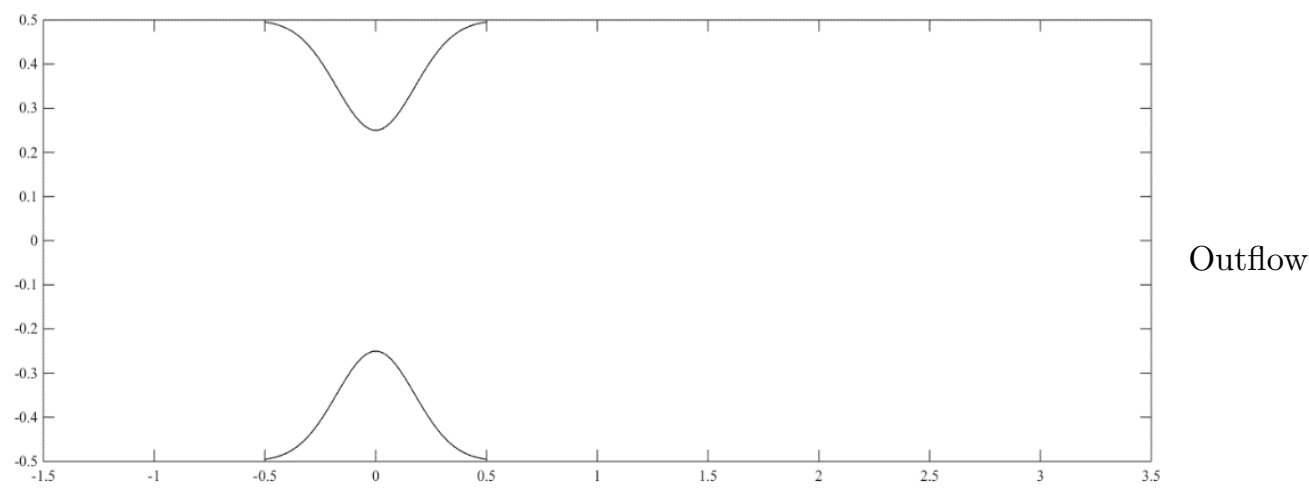

Figure 2: Geometry of Straight Rectangular Channel with 50\% Occluded Bell Shaped Stenosis

The boundary conditions are prescribed as a non-slip conditions at the walls, a parabolic velocity profiles at the inflow and a traction free condition at the outlet. In addition, the results are computed using $\operatorname{Re}=400, \rho=1050 \mathrm{kgm}^{-3}, \mu=0.0035 \mathrm{kgm}^{-1} \mathrm{~s}^{-1}, \sigma=0.8 \mathrm{Sm}^{-1}$ and are found converged with $\tau=1 \times 10^{-4}$. The results for the maximum velocity and its location as well as the pressure drop at the centre of the stenosis are validated with the results found by the previous work [19] and COMSOL multiphysics software as tabulated in Table 1.

Table 1: Results for Maximum $u$ - velocity and its Location and Pressure Drop

\begin{tabular}{|c|c|c|}
\hline & $\begin{array}{c}\text { Maximum } u \text { - velocity } \\
\text { and its location }(x, y)\end{array}$ & $\begin{array}{c}\text { Pressure drop, } \\
\nabla p \text { at } x=0\end{array}$ \\
\hline Present study & 1.5993 at $(0.286,0)$ & 0.7928 \\
\hline COMSOL multiphysics & 1.5789 at $(0.2697,-0.0002)$ & - \\
\hline Xenos and Tzirtzilakis $[19]$ & 1.5360 & 0.8000 \\
\hline
\end{tabular}

Comparison of results that are made with the previous literature and COMSOL multiphysics software shows a small distinct of maximum $u$ - velocity and its location. Meanwhile, the pressure drop evaluated from this current study shows an excellent agreement with the previous 
work. Overall, it can be concluded that the solutions are obtained in a satisfactory agreement between the previous work, COMSOL multiphysics as well as the one computed from the source code thus shows that the source code developed is working properly.

\section{$5 \quad$ Results and Discussions}

The computational domain in this present study is discretized using the unstructured linear triangular elements. This require less number for the degrees of freedom compared to the quadratic shape functions, but is sufficient to approximate solution at similar level of accuracy. The geometrical shape of bifurcated artery is constructed based on the data used from [15] and [16]: $a=0.0075 \mathrm{~m}, l_{0}=0.015 \mathrm{~m}, d=0.005 \mathrm{~m}, x_{\max }=0.06 \mathrm{~m}, x_{1}=0.025 \mathrm{~m}$ and $\tau_{m}=0.4 a$. Blood is assumed to behave as a Newtonian fluid with MHD effects by using the following set of parameters as suggested by [10] and [15]: $\rho=1050 \mathrm{kgm}^{-3}, \mu=0.0035 \mathrm{kgm}^{-1} \mathrm{~s}^{-1}$ and $\sigma=0.8 \mathrm{Sm}^{-1}$.

From Table 2, one may observe that the maximum velocities are decreasing with an increase in Hartmann and Reynolds numbers. Apparently, higher Reynolds number $(\mathrm{Re}=500)$ exhibits an accelerated blood flow and huge area of stagnating flow indicated by the contouring colours in comparison to the lower Reynolds number $(\mathrm{Re}=100)$. Meanwhile, the strength of Lorentz force produced become stronger with increase in Hartmann number that leads to retardation on the fluid's motion.

In Figure 3, the dimensionless axial velocity, $u$ for different Hartmann numbers equal to 0, 4 and 8 showing a reduction on flow's velocity as the magnetic intensity is increased along the centre of the second throat of an overlapping stenosis $(x=1)$. Somehow, the opposing motion caused by the Lorentz force is dominant only when the magnetic intensity is raised by $M=8$. This indicates that to ensure the flow along this stenotic region to be properly controlled, certain strength of magnetic intensity is required. Otherwise, there will be no significant effects of applying an external magnetic field along this constricted region.

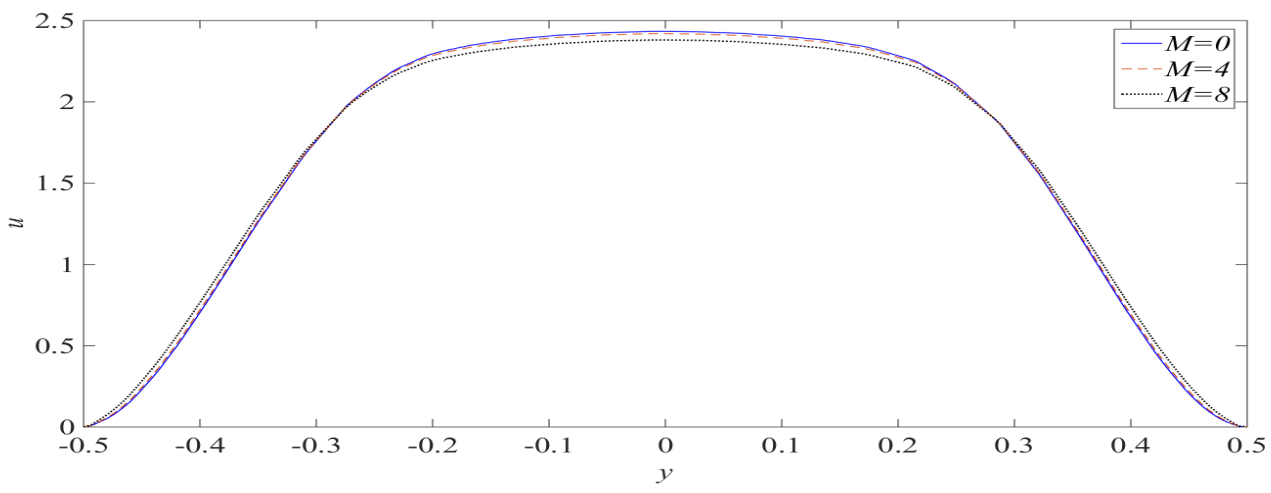

Figure 3: The Dimensionless Axial Velocity, $u$ at the Second Throat of Stenosis, $x=1$ for Different Hartmann Number $(\mathrm{Re}=300)$

Figure 4 shows the variation of axial velocity along the second throat of an overlapping stenosis, $x=1$ with different Reynolds numbers equal to 50, 200 and 500. By increasing the Reynolds number, the curves attain a flattening shape profiles and steeper velocity gradients 
Table 2: Contour of Velocity for Different Hartmann and Reynolds Numbers

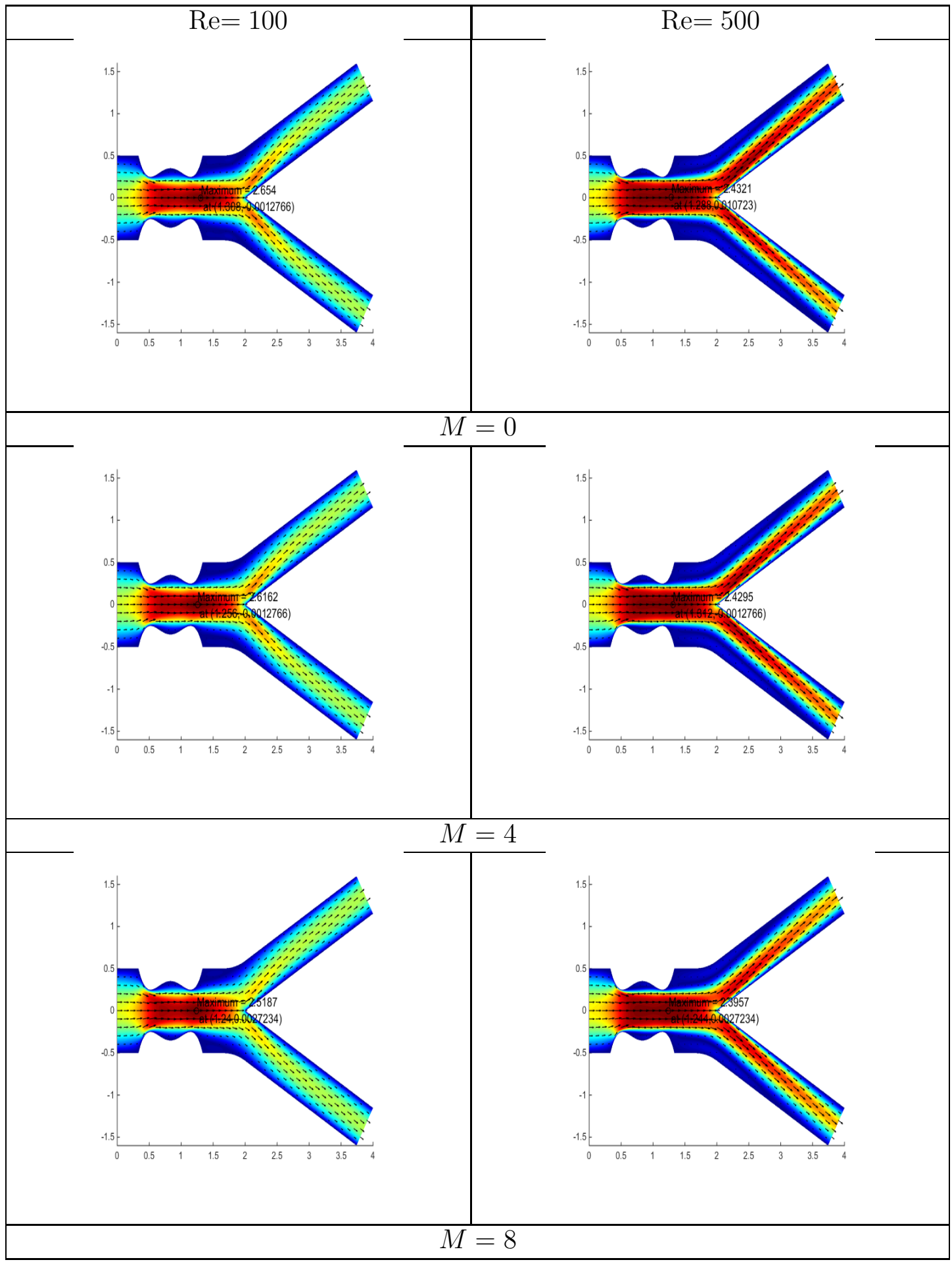


compared to the lower Reynolds number. This occurs due to the inertial forces dominated flow caused by higher velocity to overcome the viscous force. Higher Reynolds number indicates that the flow is more turbulent and more prone to develop a flow reversal along the outer wall. Thus, the velocity of fluid in an axial direction near the central axis of vessel has dropped significantly as the Reynolds number is increased from 50 to 500 which is supported by the findings from $[20]$.

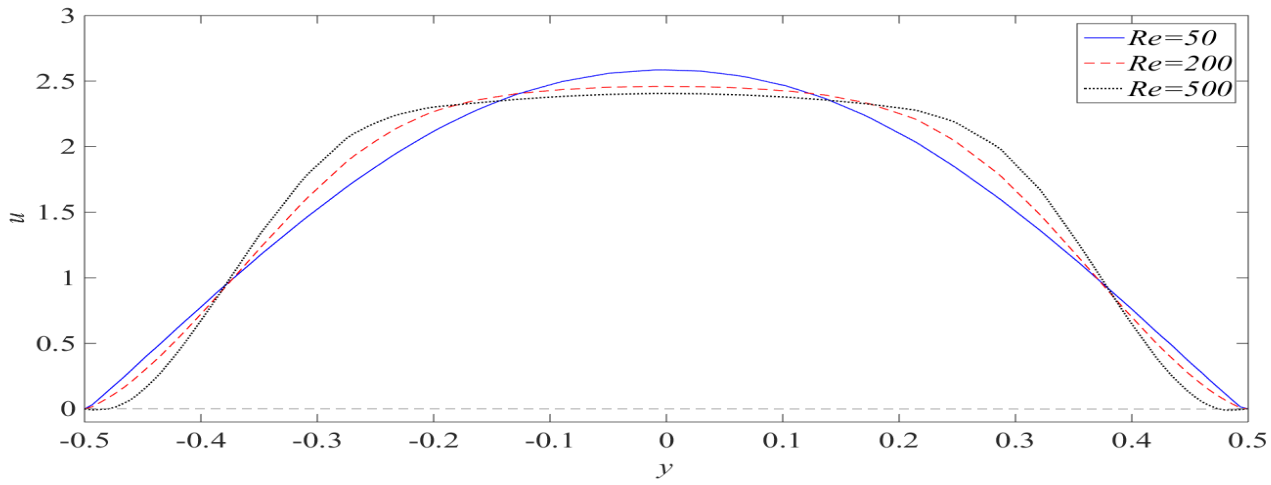

Figure 4: The Dimensionless Axial Velocity, $u$ at the Second Throat of Stenosis, $x=1$ for Different Reynolds Number $(M=2)$

The radial velocity profiles along a similar region of $x=1$ for different Hartmann numbers of 4, 8 and 12 are illustrated in Figure 5. One may observe from this current figure that the magnitudes of the radial velocity decreases with increase in Hartmann number. By comparison with the previous Figures 3 and 4, it is noticed that the magnitudes for the radial velocity appear to be much smaller than those on the axial velocity even though the sign might differ. Besides that, the magnitudes of maximum radial velocity formed near the arterial wall is also reduced considerably as the magnetic intensity is strengthen. This finding on the radial velocity profiles is found in excellent agreement as those found in [21].

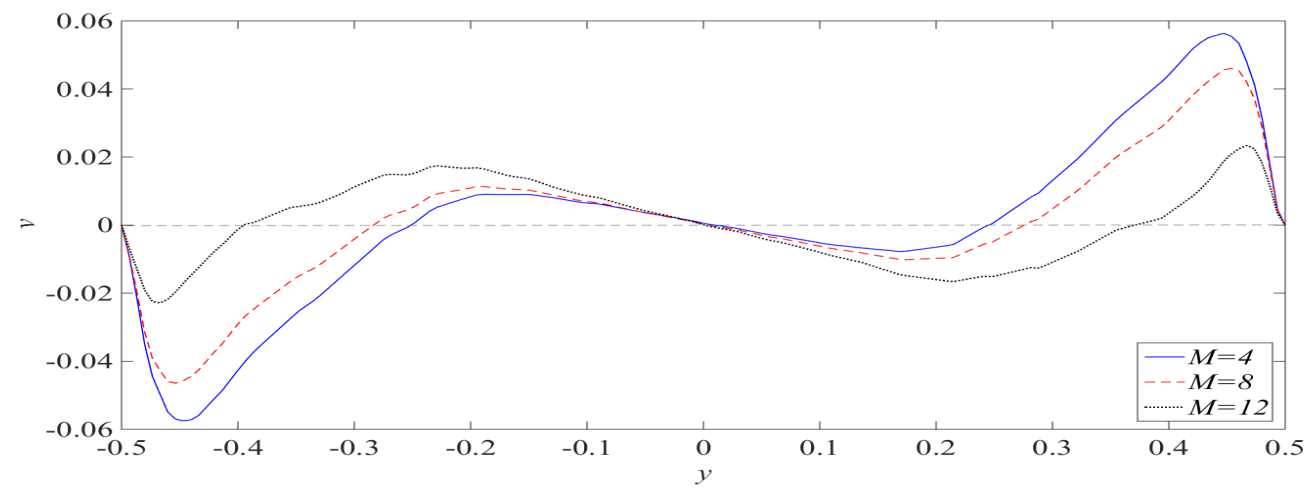

Figure 5: The Dimensionless Radial Velocity, $v$ at the Second Throat of Stenosis, $x=1$ for Different Hartmann Number $(R e=300)$

The results for the radial velocity profiles exhibited in Figure 6 for different Reynolds numbers of 50, 300 and 600 along the axial position of $x=1$ are found to be quite interesting 
to note. Obviously seen over this present figure that the profiles for lower Reynolds number $(\mathrm{Re}=50)$ behaves in a different features as the other curves with higher Reynolds number $(\mathrm{Re}=300$ and 500). In addition, it is also discovered that the radial velocity profiles with higher Reynolds number attain a smaller magnitude of peak radial velocity near the arterial wall. This shows that flows characterised by low Reynolds number (more laminar) tends to have higher axial as well as radial velocity at the stenotic region. This outcome agreed well with the one discovered by [20].

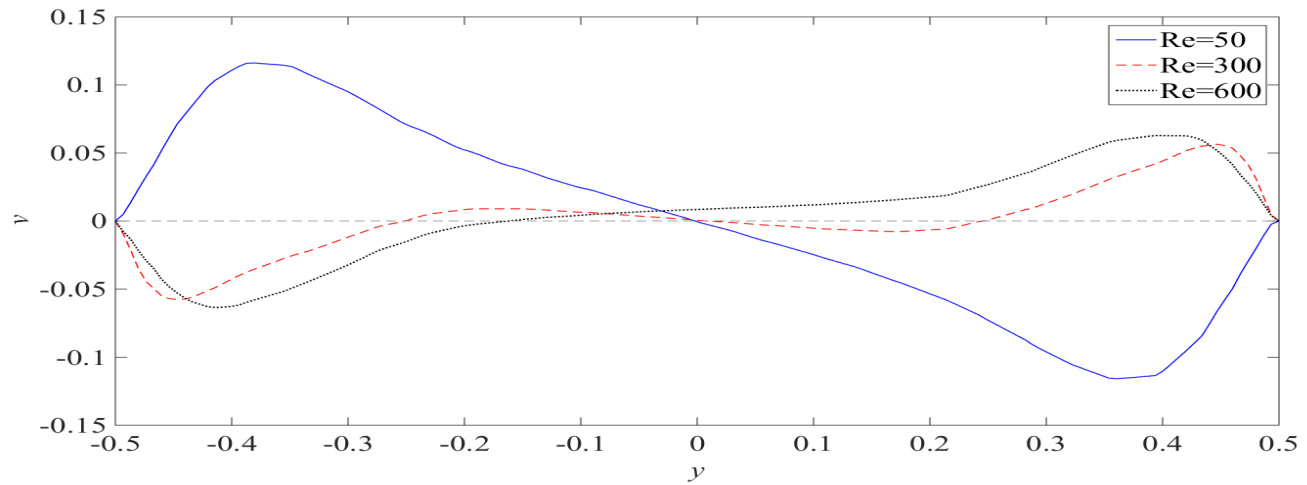

Figure 6: The Dimensionless Radial Velocity, $v$ at the Second Throat of Stenosis, $x=1$ for Different Reynolds Number $(M=4)$

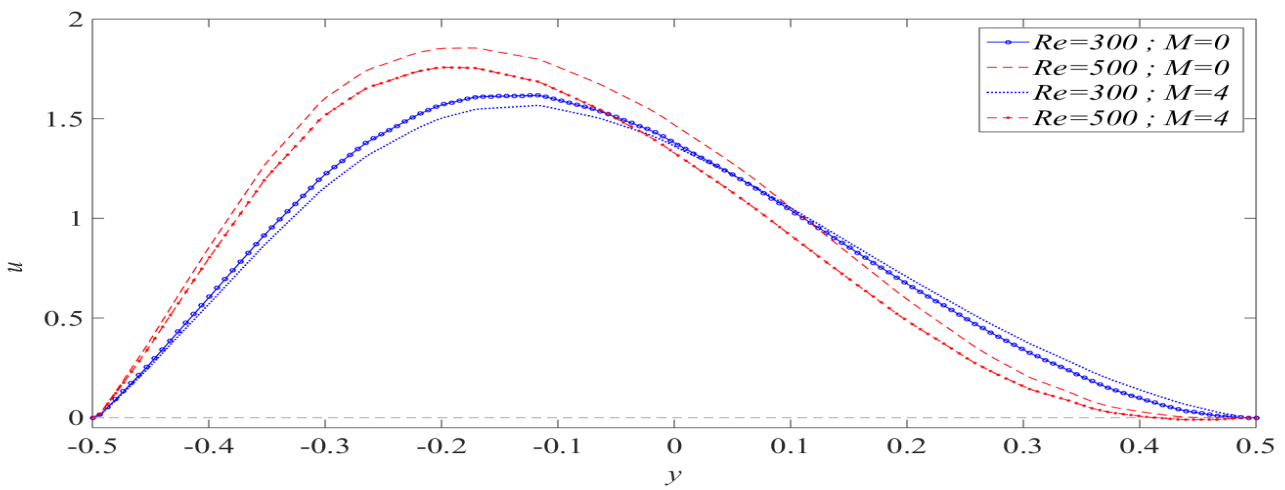

Figure 7: The Dimensionless Axial Velocity, $u$ at the Upper Daughter Branch, $x=3.0118$ for Different Hartmann and Reynolds Numbers

The variation of the axial velocity profiles for different Hartmann and Reynolds numbers at the upper daughter branch position of $x=3.0118$ are portrayed as in Figure 7 . Basically, the graph is skewed to the left because the streaming blood are just passing through a region with flow recirculation along the outer wall side that pushes the fluid layers into the inner wall side. With increasing strength of magnetic intensity, the flow at the daughter branch decelerates as well. In contrast, as the Reynolds number is increased, the flow velocity is also increases near the inner wall side but is prone to develop a negative flow near the opposite side. The negative flow that is formed near the outer wall somehow could be overcome by the application of an external magnetic field that destroys the recirculation area that is developed along the outer wall sides as shown in this figure. The magnitudes of an axial velocity at the daughter branch 
noticed to be lower than those at the stenotic region as shown in Figures 3 and 4 since part of this flow has been decelerated after it is obstructed at the stenosed part of the mother artery.

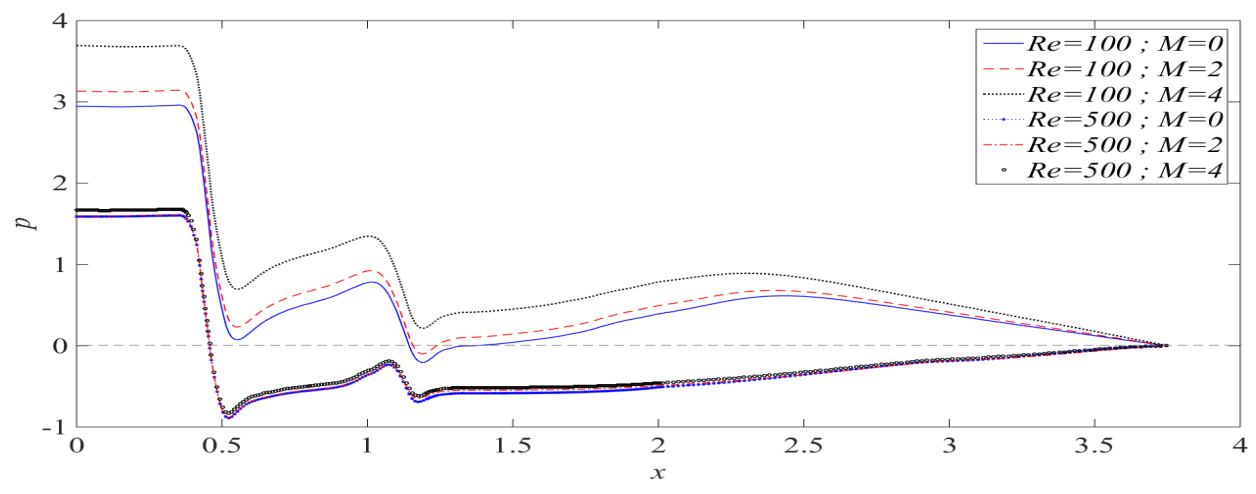

Figure 8: The Dimensionless Wall Pressure, $p$ along the Outer Wall for Different Hartmann and Reynolds Numbers

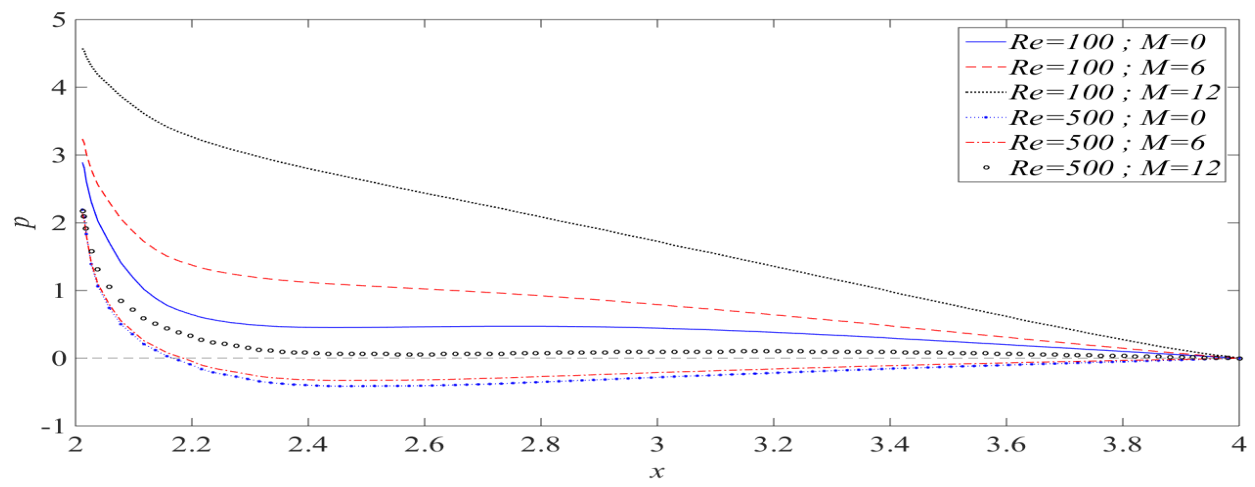

Figure 9: The Dimensionless Wall Pressure, $p$ along the Inner Wall for Different Hartmann and Reynolds Numbers

Studied on the pressure loss is very crucial since it can be an indication to adequate blood supply to the other organs or vice versa. Several plots have been made in Figures 8 and 9 to characterise the variation of wall pressure with different Hartmann and Reynolds numbers along the outer and inner wall, respectively. Since higher Reynolds number is synonym with the occurrence of eddies, vortices and tend to be related with a turbulence flow phenomenon. Hence, as can be seen from Figure 8, the wall pressure for Re $=500$ tends to have a negative wall pressure along the stenotic region and downstream of it due to the flow separation formed close to the outer wall surface. However, the effects of Hartmann number are diminished for higher Reynolds number flow since it seems that the variation between the curves are very small as indicated in Figure 8. The velocity at low Reynolds number is flowing at an increasing speed near the arterial wall, thus the magnitudes of pressure exerted along the outer arterial wall by $R e=100$ are larger and more noticeable in comparison to $\operatorname{Re}=500$.

The pressure distribution along the inner wall for both Reynolds numbers of 100 and 500 also increases with increase in Hartmann number. All the curves are gradually decreases from their respective maximum value at the apex to a zero pressure applied at the outlet. There is 
a significant variation on pressure for low Reynolds number $(\mathrm{Re}=100)$ flow as the magnetic intensity is increased. This variation gets reduced as the Reynolds number growth to 500 . Flow separation formed by a more turbulence flow $(\mathrm{Re}=500)$ close to the outer wall tends to increase the velocity at the inner wall side, thus lowering resistance to flow and at the same time exert only a small pressure between the fluid layers and the arterial wall. This resulted to the negatives valued pressure that managed to be overcome as the magnetic intensity is raised to 12 .

\section{Conclusions}

The effects of dimensionless parameters known as Hartmann and Reynolds numbers in the steady blood flow phenomena in a bifurcated artery with an overlapping stenosis at parent's arterial lumen have been presented in this study. The effects of MHD and flow motion transition from laminar to more turbulence characterised by Hartmann and Reynolds numbers on various fluid parameters like blood velocity and pressure are studied. Generally, the magnetic field reduces the blood velocity both in axial and radial directions and restricts the occurrence of flow reversal downstream of the stenotic region as the magnetic intensity is increased. Despite that, to reduce the blood's velocity substantially in an axial direction along the stenosed region, certain higher values of magnetic intensity are required. These higher magnitudes of Hartmann number are good enough to recover the negative wall pressure along the inner wall side at higher Reynolds number. These negative values pressure exerted on the wall especially the one form at the stenotic region may cause the artery to collapse due to insufficient pressure to support the opening of the arterial lumen. The MHD effects onto the wall pressure diminishes at higher Reynolds number due to the inertial forces dominated flow which resist any change in momentum. At the daughter branch, the effects of Reynolds number are more prominent than the Hartmann number influences. Somehow, the MHD effects at the daughter branch having no constriction are more obvious compared to region with $40 \%$ constriction at the mother artery. Rise in Reynolds number promote the occurrence of flow reversal, stagnation and vortex that may induce the incidence of thrombosis. This investigation would be very helpful for medical practitioners to analyse the impact of magnetic field by various magnetic therapy toward patients.

Limitations of this study involve the consideration of steady, rigid arterial wall and Newtonian nature of blood. In future, a GNL model will be included in the modelling to understand more on various physiological problems of diseased vessels.

\section{Acknowledgement}

The authors would like to acknowledge the Ministry of Education (MOE), Research Management Centre (RMC) and UniversitiTeknologi Malaysia for the financial support through vote number $19 \mathrm{H} 00$ for this research 


\section{References}

[1] Huh, H. K., Ha, H. and Lee, S. J. Effect of non-Newtonian viscosity on the fluid-dynamic characteristics in stenotic vessels. Experiments in Fluids. 2015. 56(8): 1-12.

[2] Sankar, A. R., Gunakala, S. R. and Comissiong, D. M. G. Two-layered blood flow through a composite stenosis in the presence of a magnetic field. International Journal of Application or Innovation in Engineering and Management. 2013. 2(12): 30-41.

[3] Weddell, J. C., Kwack, J. H., Imoukhuede, P. I. and Masud, A. Hemodynamic analysis in an idealized artery tree: differences in wall shear stress between Newtonian and nonNewtonian blood models. PLos ONE. 2015. 10(4): 1-23.

[4] Roy, R. and Riahi, D. N. On blood flow in an artery with an unsteady overlapping stenosis: Mathematical and computer modeling. International Journal of Fluid Mechanics Research. 2013. 40(1): 9-26.

[5] Stroud, J. S., Berger, S. A. and Saloner, D. Numerical analysis of flow through a severely stenotic carotid artery bifurcation. Journal of Biomechanical Engineering. 2002. 124(1): 9-20.

[6] Varshney, G., Katiyar, V. K. and Kumar, S. Effect of magnetic field on the blood flow in artery having multiple stenosis: A numerical study. International Journal of Engineering, Science and Technology. 2010. 2(2): 67-82.

[7] Bhatnagar, A. and Shrivastav, R. K. Analysis of MHD flow of blood through a multiple stenosed artery in the presence of slip velocity. International Journal of Innovative Research in Advanced Engineering (IJIRAE). 2014. 1(10): 250-257.

[8] Papafaklis, M. I. and Lampros, K. M. Intravascular imaging and haemodynamics: The role of shear stress in atherosclerosis and in-stent restenosis, in intravascular imaging. In:Tsakanikas, C. V. (Ed.).Current Applications and Research Developments. Hershey, PA, USA: IGI Global. 2012. 326-348.

[9] Srinivasacharya, D. and Rao, G. M. Micropolar fluid flow through a stenosed bifurcated artery. Nonlinear Analysis: Modelling and Control. 2017. 22(2): 147-159.

[10] Rabby, M. G., Shupti, S. P. and Molla, M. M. Pulsatile non-Newtonian laminar blood flows through arterial double stenoses. Journal of Fluids. 2014. 2014(757902): 1-13.

[11] Ro, K. C. and Ryou, H. S. Numerical study on turbulent blood flow in a stenosed artery bifurcation under periodic body acceleration using a modified $\mathrm{k}-\varepsilon$ model. Korea-Australia Rheology Journal. 2010. 22(2): 129-139.

[12] Tzirakis, K., Botti, L., Vavourakis, V. and Papaharilaou, Y. Numerical modeling of nonNewtonian biomagnetic fluid flow. Computers \&6 Fluids. 2015. 126(2016): 170-180.

[13] Bose, S. and Banerjee, M. Magnetic particle capture for biomagnetic fluid flow in stenosed aortic bifurcation considering particle-fluid coupling. Journal of Magnetism and Magnetic Materials. 2015. 385(2015): 32-46.

[14] Machado, F., Zinani, F. and Frey, S. Galerkin Least-Squares approximations for HerschelBulkley fluid flows through an axisymmetric abrupt expansion. 19th International Congress of Mechanical Engineering. November 5-9. Brasilis: ABCM. 2007. 1-8. 
[15] Chakravarty, S. and Mandal, P. K. An analysis of pulsatile flow in a model aortic bifurcation.International Journal of Engineering Science. 1997. 35(4): 409-422.

[16] Chakravarty, S. and Mandal, P. K. Mathematical modelling of blood flow through an overlapping arterial stenosis. Mathematical and Computer Modelling. 1994. 19(1): 59-70.

[17] Zinani, F. and Frey, S. Galerkin least-squares solutions for purely viscous flows of shearthinning fluids and regularized yield stress fluids. Journal of the Brazilian Society of Mechanical Sciences and Engineering. 2007. 29(4): 432-443.

[18] Masud, A. and Kwack, J. A stabilized mixed finite element method for the incompressible shear-rate dependent non-Newtonian fluids :variational multiscale framework and consistent linearization. Comput. Methods Appl. Mech. Engrg. 2010. 200(2011): 577-596.

[19] Xenos, M. A. and Tzirtzilakis, E. E. MHD effects on blood flow in a stenosis. Advances in Dynamical Systems and Applications. 2013. 8(2): 427-437.

[20] Tan Yan Bin. The Effects of Gravitational Acceleration on Micropolar Fluid Model of Blood Flow in a Bifurcated Stenosed Artery, Universiti Teknologi Malaysia. PhD. Thesis. 2017.

[21] Ikbal, Md. A., Chakravarty, S., Wong, K. K. L., Mazumdar, J. and Mandal, P. K. Unsteady response of non-Newtonian blood flow through a stenosed artery in magnetic field. Journal of Computational and Applied Mathematics. 2009. 230(1): 243-259. 